\title{
Identification of a peripheral blood long non-coding RNA (Upperhand) as a potential diagnostic marker of coronary artery disease
}

\author{
Xuejie $\mathrm{Li}^{1, *}$, Zhenzhou Zhao ${ }^{1, *}$, Chuanyu Gao ${ }^{1}$, Lixin $\mathrm{Rao}^{1}$, Peiyuan $\mathrm{Hao}^{1}$, \\ Dongdong Jian ${ }^{2}$, Wentao $\mathrm{Li}^{1}$, Haiyu Tang ${ }^{1}$, Muwei $\mathrm{Li}^{1}$
}

${ }^{1}$ Department of Cardiology, People's Hospital of Zhengzhou University, Zhengzhou University, Zhengzhou, China

${ }^{2}$ Department of Cardiology, The First Affiliated Hospital of Zhejiang University,

Zhejiang University, Hangzhou, China

\begin{abstract}
Background: Long non-coding RNAs (IncRNAs) have been confirmed to be involved in the pathological processes of multiple diseases. However, the characteristic expression of IncRNAs in peripheral blood of coronary artery disease (CAD) patients and whether some of these IncRNAs can be used as diagnostic biomarkers for $C A D$ requires further investigation.

Methods: Six healthy and CAD individuals were selected for microarray analysis, and 5 differentially expressed IncRNAs were selected and confirmed in the second cohort consisting of 30 control individuals and 30 CAD patients with different SYNTAX scores. Upperhand were verified in the third cohort consisting of 115 controls and 137 CAD patients.

Results: Thirty one IncRNAs were differentially expressed between the two groups, among whom, 25 were upregulated in the CAD group and 6 were downregulated. Four of the selected five IncRNAs were significantly upregulated in the CAD group, and Upperhand had the largest area under the curve (AUC). The diagnostic value of Upperhand was tested further, and it remained having a high diagnostic value. Conclusions: The expression level of Upperhand in peripheral blood of CAD patients is significantly higher than in control individuals, and is correlated with severity of CAD. Upperhand is a potential diagnostic biomarker of CAD, and when combined with TCONS_00029157, diagnostic value slightly increased. (Cardiol J 2018; 25, 3: 393-402)
\end{abstract}

Key words: coronary artery disease, long non-coding RNA, microarray analysis, biomarker

\section{Introduction}

Coronary artery disease (CAD) has a significant impact on public health. According to a 2014 statistical summary by the World Health Organization (WHO), cardiovascular diseases are the most lethal non-communicable diseases worldwide in 2012 , accounting for $46 \%$. Although there are standard treatments for CAD at present, such as drug therapy, percutaneous coronary intervention (PCI) and coronary artery bypass graft (CABG) surgery, the prognosis is still not satisfactory for some patients $[1,2]$.
This discrepancy is mainly because the current diagnostic methods cannot achieve high accuracy and convenience at the same time, which obviously increases the missed diagnosis rate. Therefore, a new highly sensitive and convenient diagnostic biomarker of CAD would be of great value.

In the past, non-coding RNAs had been considered the "dark matter" in the genome because of their indefinite types and functions. However, increasing evidence has demonstrated that though non-coding RNAs do not code for proteins directly, they do play important regulatory roles in the

Address for correspondence: Muwei Li, MD, Department of Cardiology, People's Hospital of Zhengzhou University,

Zhengzhou University, Zhengzhou, China, tel: +86-13838083966, fax: +86-0371-65580358, e-mail: limuwei@medmail.com.cn

Received: 17.02 .2017

Accepted: 11.07.2017

*These authors contributed equally to this work. 
transcription and translation of protein-coding genes. Long non-coding RNA (lncRNA) is one important type of non-coding RNAs with a length greater than 200 nucleotides. LncRNAs are able to regulate gene expression through multiple approaches, including transcriptional regulation, post-transcriptional regulation and regulation of pre-mRNA splicing. In a word, lncRNAs have a broad range of biological functions [3-7].

LncRNAs have been confirmed to be intimately involved in various diseases, such as $\mathrm{Alz}$ heimer's disease, schizophrenia, diabetes mellitus, cancer [8-12]. Lu et al. [13] found that lncRNA H19 can be used as a biomarker for the early diagnosis of gastric cancer. de Kok et al. [14] revealed that lncRNA PCA3 in urine can be applied to diagnose prostate cancer, with a higher specificity than the prostate-specific antigen (PSA) test which is now widely used in clinics. Xie et al. [15] indicated that IncRNA HULC can be utilized to diagnose hepatocellular carcinoma. In cardiovascular diseases, lncRNAs have also been verified to be involved in the pathological process of heart failure, myocardial infarction, dilated cardiomyopathy and coronary atherosclerosis [16-22]. Kumarswamy et al. [17], found, with the use of microarray analysis, that lncRNA-LIPCAR was substantially negatively related to left ventricular remodeling; the expression level of LIPCAR was positively associated with mortality of patients with systolic heart failure was also verified. Chromosome $9 \mathrm{p} 21$ contains a lot of CAD related single nucleotide polymorphisms, one of which is overlapping with antisense noncoding RNA in the INK4 locus (ANRIL). ANRIL expresses in many CAD related tissues and cells, such as coronary artery smooth muscle cells, vascular endothelial cells and atherosclerotic plaques [23]. In the present study, the peripheral blood IncRNA profiles between control individuals were compared and matched with CAD patients by microarray analysis, the findings were then tested in larger independent cohorts.

\section{Methods}

\section{Study population}

This study included a total of 324 participants, all of whom were enrolled from outpatients and inpatients of the Department of Cardiology of the People's Hospital of Zhengzhou University from July 2015 to June 2016. This study had a total of three cohorts (their clinical and demographic characteristics are shown in Tables 1-3). Subjects with any of the following characteristics were ex-
Table 1. The clinical and demographic characteristics of the first cohort.

\begin{tabular}{lccc}
\hline & $\begin{array}{c}\text { Control } \\
\text { group }\end{array}$ & $\begin{array}{c}\text { CAD } \\
\text { group }\end{array}$ & P \\
\hline Male gender & $3(50 \%)$ & $3(50 \%)$ & 1 \\
Age [years] & $60 \pm 2.3$ & $61.7 \pm 7.9$ & 0.629 \\
Hypertension & $1(16.7 \%)$ & $2(33.3 \%)$ & 1 \\
Diabetes mellitus & 0 & 0 & 1 \\
Smoker & $1(16.7 \%)$ & $1(16.7 \%)$ & 1 \\
HbA1c [\%] & $5.4 \pm 0.1$ & $5.4 \pm 0.2$ & 0.585 \\
TC [mmol/L] & $3.5 \pm 0.9$ & $4.2 \pm 0.9$ & 0.179 \\
TG [mmol/L] & $0.9 \pm 0.2$ & $1.1 \pm 0.4$ & 0.315 \\
HDL [mmol/L] & $1.3 \pm 0.3$ & $1.0 \pm 0.2$ & 0.061 \\
LDL [mmol/L] & $1.8 \pm 0.7$ & $2.1 \pm 0.5$ & 0.355 \\
ALT [U/L] & $36.3 \pm 17.8$ & $30.0 \pm 11.8$ & 0.485 \\
AST [U/L] & $23.5 \pm 3.8$ & $28.3 \pm 10.0$ & 0.293 \\
Scr $[\mu \mathrm{mol} / \mathrm{L}]$ & $58.7 \pm 3.9$ & $67.0 \pm 7.0$ & 0.029 \\
FT4I [pmol/L] & $14.4 \pm 2.7$ & $15.0 \pm 1.1$ & 0.629 \\
\hline
\end{tabular}

CAD - coronary artery disease; TC - total cholesterol; TG - triglyceride; HDL — high density lipoprotein; LDL — low density lipoprotein; ALT - alanine transaminase; AST - aspartate transaminase; Scr - serum creatinine, FT4I — free thyroxine index

Table 2. The clinical and demographic characteristics of the second cohort.

\begin{tabular}{lccc}
\hline & $\begin{array}{c}\text { Control } \\
\text { group }\end{array}$ & $\begin{array}{c}\text { CAD } \\
\text { group }\end{array}$ & P \\
\hline Male gender & $12(40 \%)$ & $17(56.7 \%)$ & 0.301 \\
Age [years] & $57.3 \pm 7.5$ & $59.3 \pm 7.1$ & 0.284 \\
Hypertension & $10(33.3 \%)$ & $16(53.3 \%)$ & 0.193 \\
Diabetes mellitus & $7(23.3 \%)$ & $13(43.3 \%)$ & 0.171 \\
Smoker & $8(26.7 \%)$ & $12(40 \%)$ & 0.411 \\
HbA1c [\%] & $5.4(5.0,5.7)$ & $5.6(5.3,6.3)$ & 0.187 \\
TC [mmol/L] & $4.0 \pm 0.7$ & $4.4 \pm 0.8$ & 0.057 \\
TG [mmol/L] & $1.9 \pm 0.8$ & $1.7 \pm 0.6$ & 0.231 \\
HDL [mmol/L] & $1.0 \pm 0.2$ & $1.1 \pm 0.2$ & 0.091 \\
LDL [mmol/L] & $2.2 \pm 0.6$ & $2.6 \pm 0.8$ & 0.052 \\
ALT [U/L] & $22.7 \pm 6.0$ & $24.9 \pm 9.3$ & 0.298 \\
AST [U/L] & $23.1 \pm 5.0$ & $21.8 \pm 6.2$ & 0.387 \\
Scr [ $\mu \mathrm{mol} / \mathrm{L}]$ & $67.6 \pm 13.3$ & $66.8 \pm 20.1$ & 0.857 \\
FT4I [pmol/L] & $14.6 \pm 1.2$ & $15.2 \pm 1.5$ & 0.083 \\
CADS & 0 & $27.8 \pm 12.5$ & $<0.001$ \\
\hline
\end{tabular}

CAD - coronary artery disease; TC - total cholesterol; TG - triglyceride; HDL - high density lipoprotein; LDL — low density lipoprotein; ALT - alanine transaminase; AST — aspartate transaminase; Scr — serum creatinine, FT4I — free thyroxine index; CADS coronary artery disease score

cluded: (i) malignant tumors, (ii) hepatic or renal dysfunction, (iii) any other clinically systemic acute 
Table 3. The clinical and demographic characteristics of the third cohort.

\begin{tabular}{lccc}
\hline & $\begin{array}{c}\text { Control } \\
\text { group }\end{array}$ & $\begin{array}{c}\text { CAD } \\
\text { group }\end{array}$ & P \\
\hline Male gender & $48(41.7 \%)$ & $73(53.3 \%)$ & 0.068 \\
Age [years] & $59.2 \pm 8.7$ & $61.4 \pm 7.3$ & 0.071 \\
Hypertension & $41(35.7 \%)$ & $59(43.1 \%)$ & 0.231 \\
Diabetes mellitus & $30(26.1 \%)$ & $49(35.8 \%)$ & 0.099 \\
Smoker & $30(26.1 \%)$ & $38(27.7 \%)$ & 0.769 \\
HbA1c [\%] & $6.0 \pm 0.9$ & $6.1 \pm 0.9$ & 0.109 \\
TC [mmol/L] & $3.5 \pm 1.3$ & $4.1 \pm 1.3$ & 0.001 \\
TG [mmol/L] & $1.3 \pm 0.5$ & $1.9 \pm 0.5$ & $<0.001$ \\
HDL [mmol/L] & $1.4 \pm 0.2$ & $1.3 \pm 0.3$ & 0.040 \\
LDL [mmol/L] & $2.7 \pm 0.6$ & $3.1 \pm 0.9$ & $<0.001$ \\
ALT [U/L] & $31.7 \pm 11.2$ & $29.6 \pm 9.4$ & 0.102 \\
AST [U/L] & $28.2 \pm 7.3$ & $28.9 \pm 9.2$ & 0.522 \\
Scr [ $\mu$ mol/L] & $64.7 \pm 14.9$ & $63.6 \pm 13.9$ & 0.548 \\
FT4I [pmol/L] & $15.7 \pm 1.7$ & $15.3 \pm 1.9$ & 0.086 \\
\hline CAD - coronary artery disease; TC - total cholesterol; TG - tri- \\
glyceride; HDL - high density lipoprotein; LDL - low density lipo- \\
protein; ALT - alanine transaminase; AST - aspartate transamin- \\
ase; Scr - serum creatinine, FT4I - free thyroxine index
\end{tabular}

or chronic inflammatory disease(s), (iv) history of acute myocardial infarction, PCI or CABG, (v) autoimmune diseases, (vi) uncontrolled hypertension, and (vii) malignant arrhythmias and valvular heart diseases.

\section{Study process}

All participants received coronary angiography (CAG) to determine whether they were healthy or had CAD. 6 CAD patients and 6 controls were selected and total RNAs were extracted from their venous blood samples for microarray analysis. The screened lncRNAs were then verified in the second cohort, which included 30 normal individuals and 30 patients with different severities of CAD (as evaluated by SYNTAX scores and expressed as the coronary artery disease score [CADS]). The lncRNA with the best diagnostic value was selected as a potential biomarker. Finally, its diagnostic value was further assessed in the third independent cohort (control group, $\mathrm{n}=115$; and CAD group, $\mathrm{n}=137$ ).

\section{Definition of CAD and collection of blood}

Coronary artery disease was defined as the stenosis degree of any coronary artery $\geq 50 \%$, diagnosed by CAG according to American College of Cardiology/American Heart Association Guidelines [24]. The stenosis degree of coronary artery was measured independently by two experienced cardiology physicians by visual observation. In the control group, CAG showed no coronary atherosclerosis; treadmill exercise test (TET) presented negative results and emission computed tomography showed no myocardial ischemia, thus coronary microvascular disease was excluded. Blood $(2 \mathrm{~mL})$ was collected from the median cubital vein of patients, and stored in ethylenediaminetetraacetic acid (EDTA) anticoagulant vacutainers. The total RNA was then extracted as soon as possible.

\section{Extraction of RNA and quantitative polymerase chain reaction (Q-PCR)}

Total RNA was extracted from $1 \mathrm{~mL}$ of whole blood using the total RNA extraction kit (Biotech, Beijing, China) strictly according to the manufacturer's instructions. The extracted RNA was dissolved in RNase-free water. The concentration and purity of RNA were determined by a NanoDrop 2000 instrument (Thermo Scientific, Waltham, MA, USA). The integrity of the RNA was detected with electrophoresis on the $1 \%$ formaldehyde-denaturing gel. Complementary DNA (cDNA) was synthesized by the PrimeScript RT reagent kit (Takara Bio, Nojihigashi, Kusatsu, Japan) according to the manufacturer's instructions. Q-PCR was conducted applying SYBR-Green Premix Ex Taq (Takara Bio, Nojihigashi, Kusatsu, Japan) and monitored with an ABI PRISM 7500 Sequence Detection System (applied Biosystems, Life Technologies, Waltham, MA, USA). The relative expression levels of 1 ncRNAs were determined by Q-PCR. The sequences of the primers used in Q-PCR are shown in Table 4.

\section{Microarray expression profiling analysis of $\operatorname{lncRNA}$}

After selecting 6 participants from the control group and the $\mathrm{CAD}$ group respectively, we extracted the total RNA of the whole blood for microarray analysis. The dephosphorylation, amplification and labeling of RNA were performed according to the manufacturer's instructions. After purification, the labeled RNA was hybridized onto the microarray (Human lncRNA array, version 4.0, CapitalBio Corp., Beijing, China); with each array containing probes interrogating approximately $41,000 \mathrm{hu}-$ man lncRNAs. The microarray data of lncRNAs were analyzed with GeneSpring software V13.0 (Agilent Technologies, Santa Clara, CA, USA). The threshold values of $\geq 2$ and $\leq-2$ fold change and a $p<0.05$ of t-test were used to select differentially expressed lncRNAs. 
Table 4. Nucleotide sequences of primers used for quantitative polymerase chain reaction.

\begin{tabular}{lccc}
\hline Target & \multicolumn{1}{c}{ Forward } & Reverse & Product length \\
\hline ENST00000512246.1 & CAGGGACACCAGTCCCTACG & ACCAGGGGAACACCGATACC & 99 \\
TCONS_00023843 & CAGTAAGGCCAGCTTTGCCA & GGTTGGAACCAACAGAAGCCA & 151 \\
NR_028044.1 & GTGCGCCATCAGGAGGAGAG & CTCCTGACCACCAGCAAGAAA & 113 \\
TCONS_00029157 & TGGTCGATGGCACAATTGCTA & GCCAGGGTGAAATTTGCGGA & 101 \\
uc003wnt.1 & CCTTGCCCTCCACCCATCTA & GAGCTGGGGAGAAAAGCAGG & 150 \\
\hline
\end{tabular}

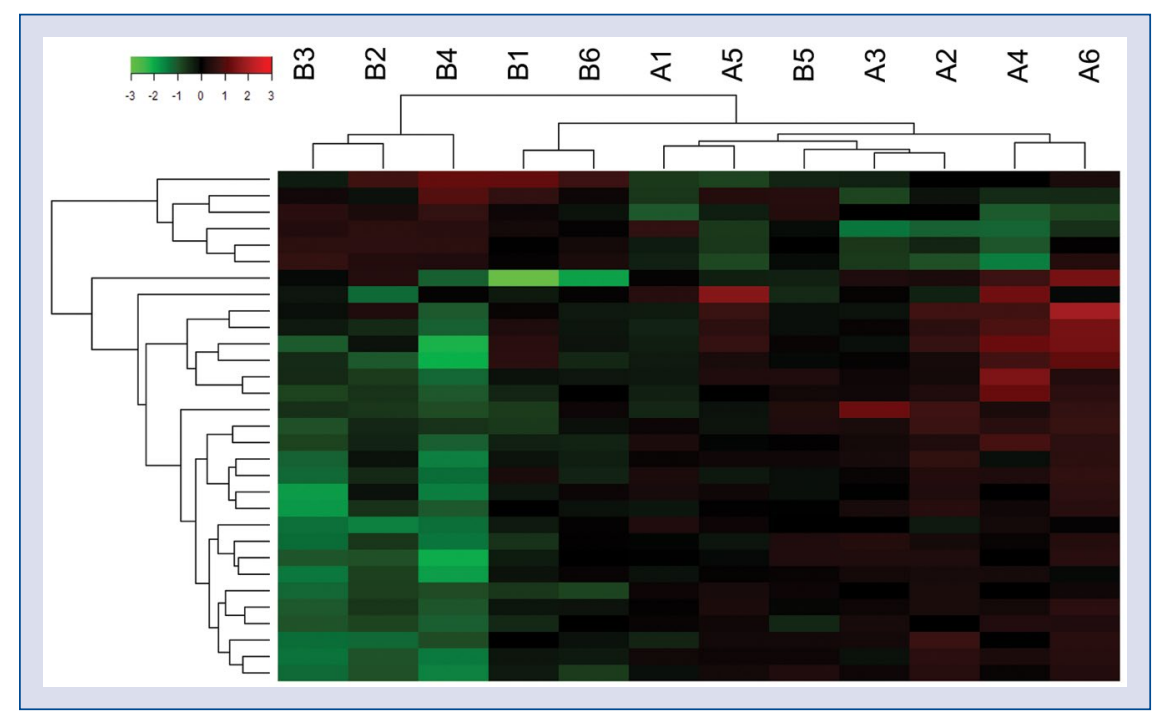

Figure 1. Heat map of long non-coding RNA (IncRNA) microarray profile. The expression of IncRNA is hierarchically clustered on the $y$-axis, and blood samples are hierarchically clustered on the $x$-axis. Expression level is presented in red and green, indicating upregulated and downregulated respectively. Numbers with A and B indicate control individuals and coronary artery disease patients.

\section{Statistical analysis}

The data in this study were expressed as means \pm standard deviations, medians (quartiles), or proportions when appropriate. In the scatterplot of the expression levels of lncRNA, the horizontal lines represent the medians. The categorical variables were verified using the $\chi^{2}$ test, and continuous variables were tested by Kolmogorov-Smirnov and Shapiro-Wilk tests to verify whether the data sets were normally distributed, and then data were analyzed by the two-tailed student t-test if they are normally distributed, or if non-normally distributed using Mann-Whitney U tests. The diagnostic value of lncRNA was evaluated by receiver operating characteristic (ROC) curve analysis. When area under the curve $(\mathrm{AUC})=0.5$, the lncRNA was defined as having no diagnostic value. The cut-off value and corresponding sensitivity and specificity were elucidated according to ROC curve analysis.
The correlations between the expression levels of $\operatorname{lncRNAs}$ and the CADS were analyzed by the Pearson correlation test. In order to calculate the odds ratio (OR), the relative expression levels of lncRNAs were multiplied by ten times to carry out logistic regression analysis. Then, OR and AUC were adjusted by introducing risk factors for CAD: smoking, hypertension, diabetes mellitus, total cholesterol (TC) and low-density lipoprotein (LDL). $\mathrm{P}<0.05$ was considered as statistically significant. All statistical analyses were conducted using SPSS 22.0 (SPSS Inc., Chicago, IL, USA).

\section{Results}

Expression profile of lncRNAs

in the whole blood of CAD patients

Results of microarray analysis demonstrated significant differences in the expression profiles 
of lncRNAs between these two groups (Fig. 1). 31 lncRNAs were differentially expressed between the two groups, of which 25 lncRNAs were upregulated and 6 were downregulated in CAD patients (Table 5). To identify the most clinically applicable biomarker, candidate biomarkers were selected in the upregulated lncRNAs utilizing strict screening criteria: $p<0.01,5 \operatorname{lncRNAs}$ were selected as candidate biomarkers: ENST00000512246.1 (referred to as Upperhand [25]), TCONS_00023843, NR_028044.1, TCONS_00029157 and uc003wnt.1 (highlighted in Table 5).

\section{Verification of the lncRNAs profile by Q-PCR}

To validate the 5 selected candidate lncRNAs, Q-PCR was performed in an independent cohort consisting of 30 control individuals (CADS $=0$, $\mathrm{n}=30$ ) and 30 CAD patients with different CADS (CADS 1-22, $\mathrm{n}=10$; CADS 23-32, $\mathrm{n}=10$; and CADS $>33, \mathrm{n}=10$ ). The results are shown in Figure 2. Four of the five candidate biomarkers were significantly upregulated in the CAD group; there was a 2.6 fold change in the expression of Upperhand, and 2.4, 2.0, and 2.1 fold changes in the expression levels of TCONS_00023843, NR_028044.1, and TCONS_0002915̄7, respectively.

\section{ROC curve analysis of the differentially expressed lncRNAs}

To test the diagnostic values of Upperhand, TCONS_00023843, NR_028044.1 and TCONS_00029157 for CAD, ROC curve analyses were carried out (Fig. 2). The AUC of Upperhand was the largest: $0.804(0.696-0.913, \mathrm{p}<0.001)$, and the cut-off value is 0.103 . The AUC of TCONS_00023843 was $0.690(0.556-0.824, \mathrm{p}=$ $=0.011)$, and the cut-off value is 0.059 . The AUCs and cut-off values were $0.739(0.612-0.866, \mathrm{p}=$ $=0.001)$ and $0.265,0.769(0.648-0.890, \mathrm{p}<0.001)$ and 0.217 for NR_028044.1 and TCONS_00029157, respectively. The sensitivity and specificity of each lncRNA were shown in Table 6.

\section{Correlations between lncRNAs and SYNTAX score for CAD}

In clinical practice, SYNTAX score is always used to measure the severity of $\mathrm{CAD}$, and on this basis, appropriate intervention methods are chosen. In this study, correlations between expression levels of candidate biomarkers and CADS were analyzed by the Pearson correlation test. Among the four chosen lncRNAs, Upperhand and
Table 5. Differentially expressed IncRNAs between coronary artery disease patients and healthy individuals.

\begin{tabular}{lcc}
\hline Upregulated IncRNAs & $\mathbf{P}$ & Fold change \\
\hline uc003wnt.1 & 0.009 & 2.0 \\
ENST00000454183.1 & 0.042 & 2.5 \\
ENST00000553211.1 & 0.036 & 2.0 \\
TCONS_00020626 & 0.029 & 2.1 \\
XR_428751.1 & 0.034 & 3.7 \\
TCONS_00015205 & 0.042 & 2.1 \\
TCONS_00023843 & 0.001 & 2.1 \\
XR_245754.1 & 0.028 & 2.1 \\
ENST00000606037.1 & 0.019 & 2.0 \\
ENST00000431705.1 & 0.049 & 2.2 \\
TCONS_00024610 & 0.035 & 2.1 \\
ENST00000416119.1 & 0.049 & 2.2 \\
NR_028044.1 & 0.002 & 2.2 \\
ENST00000539163.1 & 0.027 & 2.2 \\
ENST00000439434.1 & 0.048 & 2.0 \\
ENST00000583224.1 & 0.024 & 2.0 \\
TCONS_00013397 & 0.045 & 2.1 \\
ENST00000413810.1 & 0.011 & 2.3 \\
ENST00000512246.1 & 0.001 & 2.1 \\
TCONS_00029157 & 0.003 & 2.0 \\
ENST00000497896.1 & 0.037 & 2.1 \\
uc.342+ & 0.011 & 2.3 \\
XR_428826.1 & 0.025 & 2.0 \\
XR_428901.1 & 0.029 & 2.0 \\
ENST00000455229.1 & 0.045 & 2.8 \\
\hline Downregulated IncRNAs & $\mathbf{P}$ & Fold change \\
\hline ENST00000451350.1 & 0.009 & 2.4 \\
XR_426864.1 & 0.037 & 2.1 \\
RNA33481 & 0.014 & 2.0 \\
TCONS_00017757 & 0.010 & 2.5 \\
\hline RNA147187 & 0.005 & 2.2 \\
\hline & 0.002 & 2.1 \\
\hline
\end{tabular}

TCONS_00029157 were correlated with the CADS. Based on the AUCs of the candidates and their correlations with the CADS, Upperhand was chosen as the potential biomarker for the diagnosis of CAD.

Further clinical validation of the biomarker

The diagnostic value of Upperhand was verified in another independent cohort (control group, $\mathrm{n}=115$; and CAD group, $\mathrm{n}=137$ ). Results showed that the expression level of Upperhand was evidently upregulated in the CAD group, with 


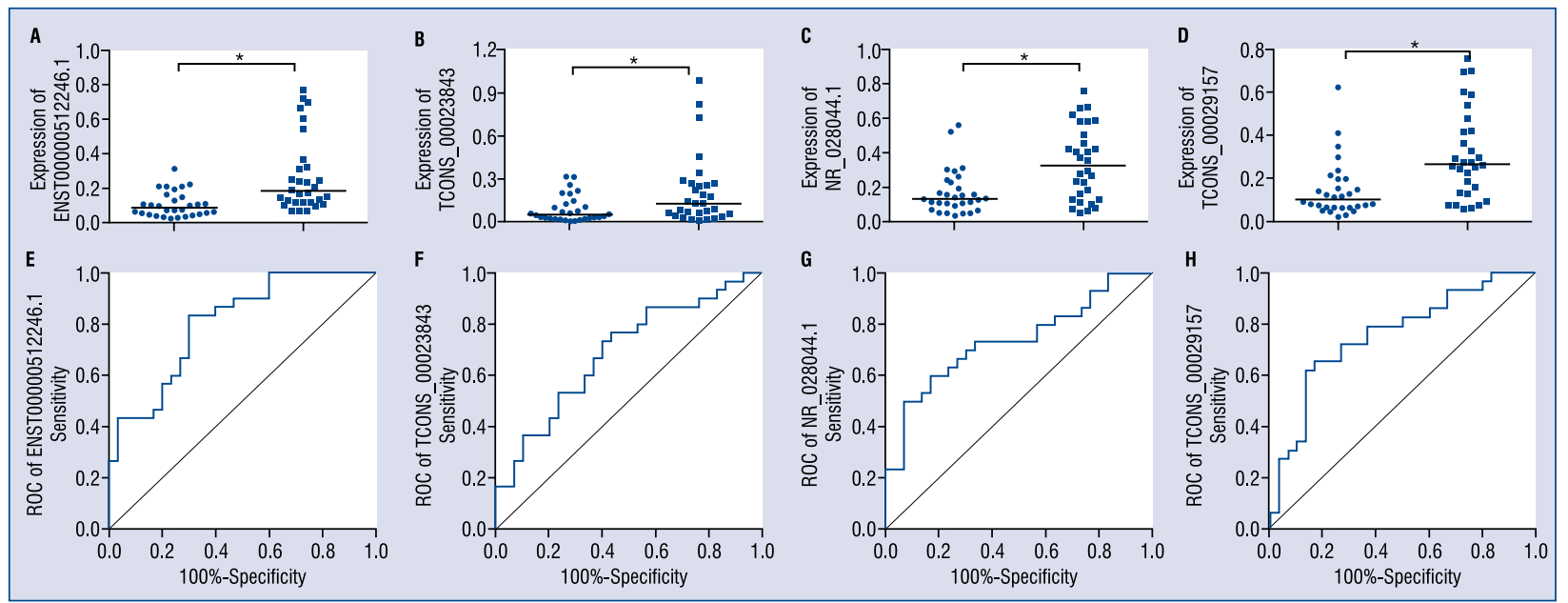

Figure 2. Expression levels of selected long non-coding RNA (IncRNA) quantified by quantitative polymerase chain reaction. Panels A-D indicate the expression levels of Upperhand, TCONS_00023843, NR_028044.1 and TCONS_00029157 in the control group and coronary artery disease group, ${ }^{*} p<0.05$; Panels E-H indicate the receiver operating characteristic curve analyses of the above mentioned IncRNAs for the diagnoses of coronary artery disease.

Table 6. The sensitivity and specificity of the selected long non-coding RNA (IncRNA).

\begin{tabular}{lccccc}
\hline & AUC & $95 \% \mathrm{Cl}$ & Sensitivity & Specificity & P \\
\hline ENST00000512246.1 & 0.804 & $0.696-0.913$ & 0.833 & 0.7 & $<0.001$ \\
TCONS_00023843 & 0.69 & $0.556-0.824$ & 0.767 & 0.567 & 0.011 \\
NR_028044.1 & 0.739 & $0.612-0.866$ & 0.6 & 0.833 & 0.001 \\
TCONS_00029157 & 0.769 & $0.648-0.890$ & 0.667 & 0.833 & $<0.001$ \\
\hline
\end{tabular}

AUC - area under the curve; $\mathrm{Cl}$ - confidence interval

a 2.2 fold change (Fig. 3). The ROC curve analysis presented an AUC of $0.728(0.666-0.791, \mathrm{p}<0.001)$, with a sensitivity of 0.737 and specificity of 0.652 ; the crude OR and cut-off value was 1.64 (1.343$2.002, \mathrm{p}<0.001$ ) and 0.084 . After introducing risk factors of CAD (diabetes mellitus, smoking, hypertension, TC and LDL), the AUC slightly increased to $0.790(0.735-0.846, \mathrm{p}<0.001)$, with the sensitivity of 0.65 and specificity of 0.835 ; the adjusted OR of $1.76(1.402-2.210, \mathrm{p}<0.001)$ and cut-off value of 0.584 . The results indicated that Upperhand could serve as a diagnostic biomarker of CAD.

\section{Expression of biomarker in different genders and ages}

To investigate the expression level of Upperhand in different gender and age groups, the two groups in the third cohort was further divided according to gender and age (cut-off: 60 years old), respectively. As shown in Table 7, the distribution of Upperhand in different gender and age groups presented no statistically significant differences.

\section{Improvement in the diagnostic value}

To improve the diagnostic value of the biomarker, TCONS_00029157 was introduced. As shown in the Figure 3, TCONS_00029157 was significantly upregulated in the $\mathrm{CA} D$ group, with a change of 1.8 fold. Upperhand and TCONS_00029157 were combined, and the combination as a new biomarker was tested by ROC analysis. The results showed AUC was $0.774(0.716-0.831, \mathrm{p}<0.001)$, and the sensitivity was 0.847 and specificity was 0.6 , respectively; the cut-off value was 0.395 . After introducing the risk factors for CAD (smoking, hypertension, diabetes mellitus, LDL and TC) the AUC slightly increased to $0.846(0.798-0.893, \mathrm{p}<0.001)$, the sensitivity was 0.723 and the specificity was 0.817 ; the cut-off value was 0.546 . This implied that, compared with Upperhand, the combination of 


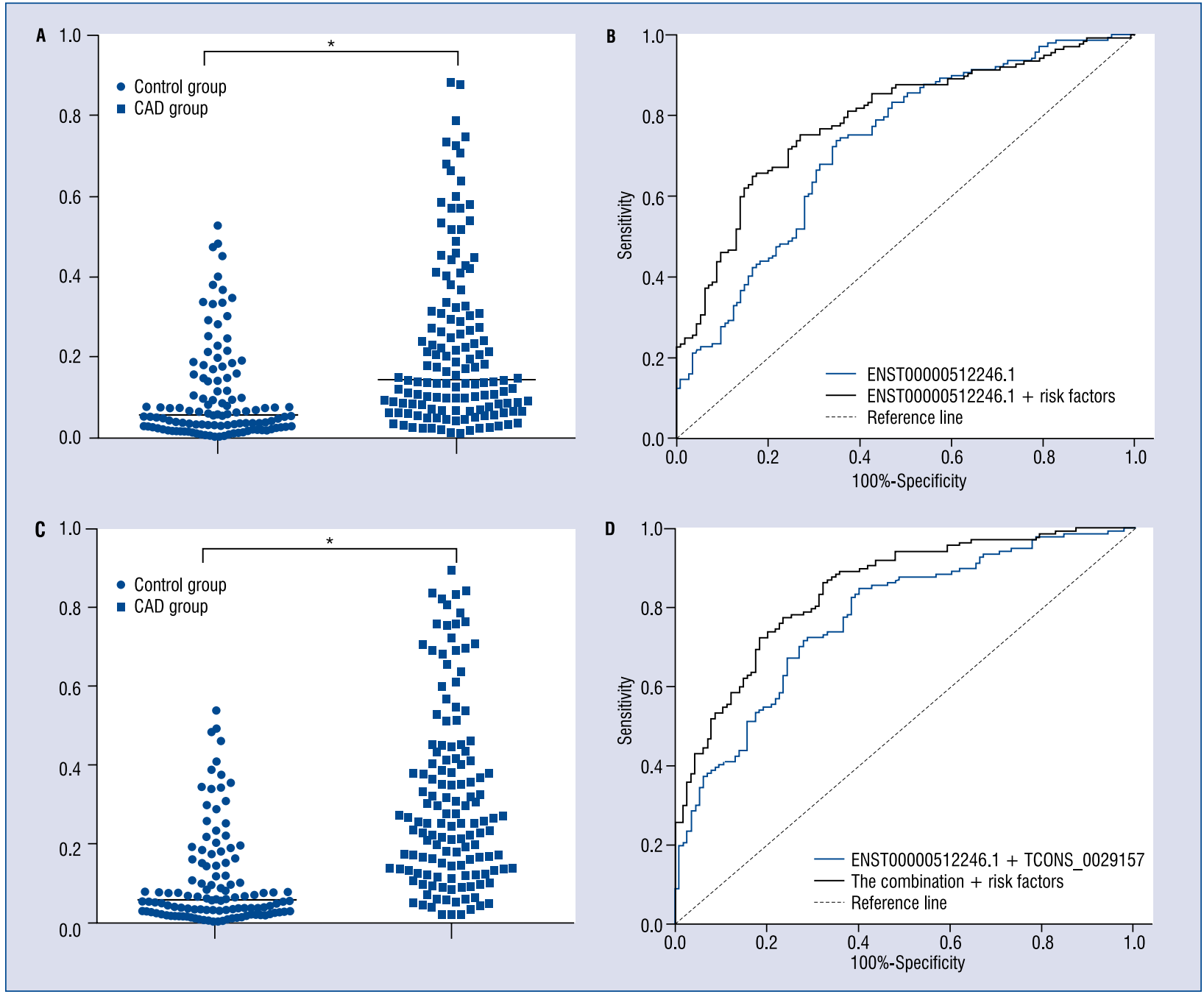

Figure 3. Expression levels of Upperhand. Panels $\mathbf{A}$ and $\mathbf{C}$ indicate the expression levels of Upperhand and Upperhand combined with TCONS_00029157 in the control group and the coronary artery disease group, respectively, ${ }^{*} p<0.05$; Panel B indicates the ROC curve analyses of Upperhand and Upperhand with risk factors for the diagnosis of coronary artery disease; Panel $\mathbf{D}$ indicates the receiver operating characteristic curve analyses of the combination long non-coding RNA (IncRNA).

Table 7. The expression levels of Upperhand in populations with different gender and age.

\begin{tabular}{|c|c|c|c|c|c|c|}
\hline Variables & Amount & $\begin{array}{l}\text { Control group } \\
\text { relative expression }\end{array}$ & $\mathbf{P}$ & Amount & $\begin{array}{l}\text { CAD group } \\
\text { relative expression }\end{array}$ & $\mathbf{P}$ \\
\hline \multicolumn{7}{|l|}{ Gender: } \\
\hline Male & 48 & $0.05(0.02,0.16)$ & \multirow{2}{*}{0.671} & 73 & $0.16(0.07,0.31)$ & \multirow{2}{*}{0.806} \\
\hline Female & 67 & $0.06(0.03,0.15)$ & & 64 & $0.14(0.07,0.33)$ & \\
\hline \multicolumn{7}{|c|}{ Age [years]: } \\
\hline$>60$ & 52 & $0.06(0.03,0.14)$ & \multirow{2}{*}{0.507} & 74 & $0.18(0.09,0.35)$ & \multirow{2}{*}{0.066} \\
\hline$\leq 60$ & 63 & $0.05(0.03,0.17)$ & & 63 & $0.14(0.06,0.29)$ & \\
\hline
\end{tabular}

CAD - coronary artery disease 
Upperhand and TCONS_00029157 as a biomarker has a higher diagnostic value for CAD.

\section{Discussion}

Due to its high morbidity and mortality worldwide, $C A D$ is a serious threat to human health. The current diagnostic methods for $\mathrm{CAD}$ are classified into two types, invasive and non-invasive. The non-invasive examinations include electrocardiogram (ECG), TET, Holter monitoring and coronary computed tomography angiography (CTA). Among them, ECG presents poor sensitivity and specificity in the diagnosis of CAD. Holter monitoring can diagnose CAD only when it captures the dynamic changes during the onset of angina. Many elderly people and patients with limited physical activities are unable to complete TET; moreover, the induction of myocardial ischemia may lead to the rupture of unstable plaques in a small number of patients, causing adverse cardiac events. In addition, coronary CTA has a relatively high cost. Invasive examinations include CAG and intravascular ultrasound (IVUS). CAG is the gold standard for the diagnosis of CAD; IVUS, in which a miniature ultrasound probe is sent into the vessel lumen to obtain a tomographic scan andcan accurately and intuitively determine residual stenosis and guide stenting [26]. In China, however, many low-income families cannot easily afford these examinations, and some patients with mild symptoms and conservative ideologies are not willing to do these invasive examinations. Therefore, a clinical examination with low cost, high accuracy and convenience is needed to facilitate the detection of CAD.

Because of the convenient sampling and low cost, hematological markers play an important role in the diagnosis of many diseases. A number of studies indicate that miRNAs are involved in the occurrence and development of various cardiovascular diseases including arrhythmia, hypertension, coronary artery calcification, myocardial hypertrophy and $\mathrm{CAD}$ [27-32]. LncRNAs could regulate gene expression by interacting with miRNAs [33, 34]. Compared with miRNAs, the mechanisms of lncRNAs in gene regulation are more complex, and the regulatory modes are more flexible [35]. Furthermore, multiple studies have shown that IncRNAs in body fluid have excellent stability [36]. Therefore, it was speculated that, compared with miRNAs, 1ncRNAs may be more suitable in serving as diagnostic biomarkers.

The result of gene ontology enrichment analysis suggests that Upperhand is correlated with LDL receptor activity, vascular smooth muscle contrac- tion and other cellular processes. It is well known that these processes all play important roles in the progression of CAD. Therefore, it was speculated that Upperhand may be involved in the progression of $\mathrm{CAD}$ through these biological processes.

At present, many methods are used to diagnose CAD besides CAG, such as ECG, Holter, TET and CTA. The sensitivity and specificity of ECG are 0.414 and 0.675 [37]. The sensitivity and specificity of Holter are 0.649 and 0.894 [38]. The sensitivity and specificity of TET are 0.650 and 0.580 [39]. The sensitivity and specificity of CTA are 0.930 and 0.860 [39]. The sensitivity and specificity of Upperhand are 0.737 and 0.652 ; when Upperhand was combined with TCONS_00029157, sensitivity and specificity of the combined biomarker were 0.847 and 0.6 , respectively. Based on this comparison, it was thought that the diagnostic value of Upperhand and the combination of Upperhand and TCONS_00029157 are greater than ECG and TET, slightly lower than Holter, while significantly lower than CTA. However, when considering the convenience and cost of diagnostic methods, it was believed that the biomarker will improve the situation of the diagnosis of CAD significantly. Furthermore, the expression characteristics of Upperhand showed no differences between populations with different genders and ages. These findings suggest that Upperhand, indeed, has the potential to serve as a diagnostic biomarker of CAD.

\section{Limitations of the study}

This study was a single-center study, with a high geographic concentration of the subjects. Therefore, the populations of other regions and countries remain to be identified in determining whether they have similar lncRNAs expression features. The lncRNAs in this study are mainly from peripheral blood mononuclear cells, so their biological link with CAD is rather weak. Meanwhile, there are differences between CAD and control individuals in pharmacotherapy, and that could also have influenced the results.

\section{Conclusions}

To sum up, this study investigates the lncRNA profile in peripheral blood of CAD patients, determines its correlation with the severity of CAD and tests the potential of lncRNA as a diagnostic biomarker of CAD. The biomarker identified in this study (Upperhand) can be tested using peripheral blood at a relatively low cost, yet specificity and sensitivity are relatively high, and the diagnos- 
tic value is slightly increased after introducing TCONS_00029157, making Upperhand a powerful tool in the diagnosis of CAD.

\section{Acknowledgements}

This study was approved by the Ethics Committee of the People's Hospital of Zhengzhou University, and all participants signed informed consent. This study was conducted in compliance with the ethical guidelines of 1975 Declaration of Helsinki. This work is supported by the Department of Science and Technology of Henan province (grant number: 122102310620).

\section{Conflict of interest: None declared}

\section{References}

1. Acharya D, Gulack BC, Loyaga-Rendon RY, et al. Clinical characteristics and outcomes of patients with myocardial infarction and cardiogenic shock undergoing coronary artery bypass surgery: data from the society of thoracic surgeons national database. Ann Thorac Surg. 2016; 101(2): 558-566, doi: 10.1016/j.athoracsur.2015.10.051, indexed in Pubmed: 26718859.

2. Waldo SW, Secemsky EA, O'Brien C, et al. Response to letter regarding article, "surgical ineligibility and mortality among patients with unprotected left main or multivessel coronary artery disease undergoing percutaneous coronary intervention". Circulation. 2015; 132(12): e156, doi:10.1161/CIRCULATIONAHA.115.016777, indexed in Pubmed: 26391303.

3. Mercer TR, Mattick JS. Structure and function of long noncoding RNAs in epigenetic regulation. Nat Struct Mol Biol. 2013; 20(3): 300-307, doi:10.1038/nsmb.2480, indexed in Pubmed: 23463315.

4. Tripathi V, Ellis JD, Shen Z, et al. The nuclear-retained noncoding RNA MALAT1 regulates alternative splicing by modulating SR splicing factor phosphorylation. Mol Cell. 2010; 39(6): 925-938, doi: 10.1016/j.molcel.2010.08.011, indexed in Pubmed: 20797886.

5. Yoon JH, Abdelmohsen K, Srikantan S, et al. LincRNA-p21 suppresses target mRNA translation. Mol Cell. 2012; 47(4): 648-655, doi:10.1016/j.molcel.2012.06.027, indexed in Pubmed: 22841487.

6. Hu W, Yuan B, Flygare J, et al. Long noncoding RNA-mediated anti-apoptotic activity in murine erythroid terminal differentiation. Genes Dev. 2011; 25(24): 2573-2578, doi: 10.1101/ gad.178780.111, indexed in Pubmed: 22155924.

7. Guttman M, Donaghey J, Carey BW, et al. lincRNAs act in the circuitry controlling pluripotency and differentiation. Nature. 2011; 477(7364): 295-300, doi: 10.1038/nature10398, indexed in Pubmed: 21874018.

8. Harries LW. Long non-coding RNAs and human disease. Biochem Soc Trans. 2012; 40(4): 902-906, doi: 10.1042/BST20120020, indexed in Pubmed:22817756.

9. Kitagawa M, Kotake Y, Ohhata T. Long non-coding RNAs involved in cancer development and cell fate determination. Curr Drug Targets. 2012; 13(13): 1616-1621, indexed in Pubmed: 22974399.

10. Chubb JE, Bradshaw NJ, Soares DC, et al. The DISC locus in psychiatric illness. Mol Psychiatry. 2008; 13(1): 36-64, doi: 10.1038/ sj.mp.4002106, indexed in Pubmed: 17912248.
11. van de Vondervoort II, Gordebeke PM, Khoshab N, et al. Long non-coding RNAs in neurodevelopmental disorders. Front Mol Neurosci. 2013; 6: 53, doi:10.3389/fnmol.2013.00053, indexed in Pubmed: 24415997.

12. Liu JY, Yao J, Li XM, et al. Pathogenic role of lncRNA-MALAT1 in endothelial cell dysfunction in diabetes mellitus. Cell Death Dis. 2014; 5: e1506, doi:10.1038/cddis.2014.466, indexed in Pubmed: 25356875 .

13. Lu T, Cui L, Zhou Y, et al. Transcriptome-wide investigation of circular RNAs in rice. RNA. 2015; 21(12): 2076-2087, doi: 10.1261/rna.052282.115, indexed in Pubmed: 26464523.

14. de Kok JB, Verhaegh GW, Roelofs RW, et al. DD3(PCA3), a very sensitive and specific marker to detect prostate tumors. Cancer Res. 2002; 62(9): 2695-2698, indexed in Pubmed: 11980670.

15. Xie H, Ma H, Zhou D. Plasma HULC as a promising novel biomarker for the detection of hepatocellular carcinoma. Biomed Res Int. 2013; 2013: 136106, doi: 10.1155/2013/136106, indexed in Pubmed: 23762823.

16. Korostowski L, Sedlak N, Engel N. The Kcnq1ot1 long noncoding RNA affects chromatin conformation and expression of $\mathrm{Kcnq1}$, but does not regulate its imprinting in the developing heart. PLoS Genet. 2012; 8(9): e1002956, doi: 10.1371/journal. pgen.1002956, indexed in Pubmed: 23028363.

17. Kumarswamy R, Bauters C, Volkmann I, et al. Circulating long noncoding RNA, LIPCAR, predicts survival in patients with heart failure. Circ Res. 2014; 114(10): 1569-1575, doi: 10.1161/ CIRCRESAHA.114.303915, indexed in Pubmed: 24663402.

18. Friedrichs F, Zugck C, Rauch GJ, et al. HBEGF, SRA1, and IK: Three cosegregating genes as determinants of cardiomyopathy. Genome Res. 2009; 19(3): 395-403, doi: 10.1101/gr.076653.108, indexed in Pubmed: 19064678.

19. Motterle A, Pu X, Wood H, et al. Functional analyses of coronary artery disease associated variation on chromosome $9 \mathrm{p} 21$ in vascular smooth muscle cells. Hum Mol Genet. 2012; 21(18): 4021-4029, doi: 10.1093/hmg/dds224, indexed in Pubmed: 22706276.

20. Holdt LM, Hoffmann S, Sass K, et al. Alu elements in ANRIL non-coding RNA at chromosome 9p21 modulate atherogenic cell functions through trans-regulation of gene networks. PLoS Genet. 2013; 9(7): e1003588, doi: 10.1371/journal.pgen.1003588, indexed in Pubmed: 23861667.

21. Bai Y, Nie S, Jiang G, et al. Regulation of CARD8 expression by ANRIL and association of CARD8 single nucleotide polymorphism rs2043211 (p.C10X) with ischemic stroke. Stroke. 2014; 45(2): 383-388, doi: 10.1161/STROKEAHA.113.003393, indexed in Pubmed: 24385277.

22. Schiano C, Costa V, Aprile M, et al. Heart failure: Pilot transcriptomic analysis of cardiac tissue by RNA-sequencing. Cardiol J. 2017; 24(5): 539-553, doi: 10.5603/CJ.a2017.0052, indexed in Pubmed: 28497843.

23. Holdt LM, Hoffmann S, Sass K, et al. ANRIL expression is associated with atherosclerosis risk at chromosome 9p21. Arterioscler Thromb Vasc Biol. 2010; 30(3): 620-627, doi: 10.1161/ ATVBAHA.109.196832, indexed in Pubmed: 20056914.

24. Scanlon PJ, Faxon DP, Audet AM, et al. ACC/AHA guidelines for coronary angiography: executive summary and recommendations. A report of the American College of Cardiology/American Heart Association Task Force on Practice Guidelines (Committee on Coronary Angiography) developed in collaboration with the Society for Cardiac Angiography and Interventions. Circulation. 1999; 99(17): 2345-2357, indexed in Pubmed: 10226103. 
25. Anderson KM, Anderson DM, McAnally JR, et al. Transcription of the non-coding RNA upperhand controls Hand2 expression and heart development. Nature. 2016; 539(7629): 433-436, doi: 10.1038/nature20128, indexed in Pubmed: 27783597.

26. Hitchner E, Zayed M, Varu V, et al. A prospective evaluation of using IVUS during percutaneous superficial femoral artery interventions. Ann Vasc Surg. 2015; 29(1): 28-33, doi: 10.1016/j. avsg.2014.07.026, indexed in Pubmed: 25194552.

27. Lu Y, Zhang Y, Wang N, et al. MicroRNA-328 contributes to adverse electrical remodeling in atrial fibrillation. Circulation. 2010; 122(23): 2378-2387, doi: 10.1161/CIRCULATIONAHA.110.958967, indexed in Pubmed: 21098446.

28. Li S, Zhu J, Zhang W, et al. Signature microRNA expression profile of essential hypertension and its novel link to human cytomegalovirus infection. Circulation. 2011; 124(2): 175-184, doi: 10.1161/ CIRCULATIONAHA.110.012237, indexed in Pubmed: 21690488.

29. Liu W, Ling S, Sun W, et al. Circulating microRNAs correlated with the level of coronary artery calcification in symptomatic patients. Sci Rep. 2015; 5: 16099, doi: 10.1038/srep16099, indexed in Pubmed: 26537670.

30. Sayed D, Hong C, Chen IY, et al. MicroRNAs play an essential role in the development of cardiac hypertrophy. Circ Res. 2007; 100(3): 416-424, doi: 10.1161/01.RES.0000257913.42552.23, indexed in Pubmed: 17234972.

31. Meder B, Keller A, Vogel B, et al. MicroRNA signatures in total peripheral blood as novel biomarkers for acute myocardial infarction. Basic Res Cardiol. 2011; 106(1): 13-23, doi: 10.1007/ s00395-010-0123-2, indexed in Pubmed: 20886220.
32. Liu Z, Zhou C, Liu Y, et al. The expression levels of plasma micoRNAs in atrial fibrillation patients. PLoS One. 2012; 7(9): e44906, doi: 10.1371/journal.pone.0044906, indexed in Pubmed: 23028671.

33. Hung T, Wang Y, Lin MF, et al. Extensive and coordinated transcription of noncoding RNAs within cell-cycle promoters. Nat Genet. 2011; 43(7): 621-629, doi: 10.1038/ng.848, indexed in Pubmed: 21642992.

34. Tay Y, Rinn J, Pandolfi PP. The multilayered complexity of ceRNA crosstalk and competition. Nature. 2014; 505(7483): 344-352, doi: 10.1038/nature12986, indexed in Pubmed: 24429633.

35. Wang KC, Chang HY. Molecular mechanisms of long noncoding RNAs. Mol Cell. 2011; 43(6): 904-914, doi: 10.1016/j.molcel.2011.08.018, indexed in Pubmed: 21925379.

36. Yang Y, Cai Y, Wu G, et al. Plasma long non-coding RNA, CoroMarker, a novel biomarker for diagnosis of coronary artery disease. Clinical Science. 2015; 129(8): 675-685, doi: 10.1042/cs20150121.

37. Tang DP, Liu LH. The relationship between ECG ST-T changes and the coronary artery disease. Chinese Medical Journal of Metallurgical Industry. 2009.

38. Jiang Y, Tian JP, Wang H, et al. Diagnostic value of combined parameters derived from ambulatory electrocardiography for detecting coronary artery disease in non-active chest pain patients. Pak J Med Sci. 2014; 30(6): 1331-1335, doi: 10.12669/ pjms.306.5176, indexed in Pubmed: 25674134.

39. Zhang J, Wang Y, Guo D. Treadmill exercise test versus coronary computed tomography angiography in diagnosis of coronary atherosclerotic heart disease: Meta-analysis. Chin J Interv Imaging Ther. 2016; 13: 562-566. 